\title{
A pandemia do COVID-19 e um novo problema de saúde pública: Os impactos emocionais em profissionais de saúde
}

The COVID-19 pandemic and a new public health problem: The emotional impacts on health professionals

La pandemia del COVID-19 y um nuevo problema de salud pública: Los impactos emocionales em los profesionales de la salud

Bárbara Fernandes de Meneses Brito ORCID: https://orcid.org/0000-0001-6477-8506 Centro Universitário Uninovafapi, Brasil E-mail: barbarafernandesmb@gmail.com

Andressa Mendes de Sousa ORCID: https://orcid.org/0000-0002-5120-9280 Centro Universitário Uninovafapi, Brasil

E-mail: andressaams18@gmail.com

Kallianny Caetano Alencar ORCID: https://orcid.org/0000-0001-7860-155X Centro Universitário Uninovafapi, Brasil E-mail: kallianny-caetano@hotmail.com

Rafaela Miranda Martins ORCID: https://orcid.org/0000-0003-3424-7952 Centro Universitário Uninovafapi, Brasil E-mail: rafaelamm1996@gmail.com

Renata Lopes Fonseca de Azevedo ORCID: https://orcid.org/0000-0003-4854-1044 Centro Universitário Uninovafapi, Brasil E-mail: renata.lfazevedo@ hotmail.com Jéssica Maírla Neves de Araújo ORCID: https://orcid.org/0000-0002-4501-0267 Centro Universitário Uninovafapi, Brasil E-mail: j.mairla@gmail.com

\begin{abstract}
Resumo
O presente trabalho visa refletir acerca da saúde mental dos profissionais atuantes na linha frente e, com isso, angariar discussões sobre os impactos emocionais na vida dos mesmos e da sociedade como resultado da pandemia de COVID-19. O estudo compreende uma revisão integrativa da literatura. As buscas por publicações foram realizadas em quatro bases de dados bibliográficas — PubMed, Biblioteca Virtual em Saúde (BVS), Scientific Eletronic Library Online (SciELO) e Literatura Latino-Americana e do Caribe em Ciências da Saúde (LILACS). Foram selecionados 16 artigos publicados entre os anos de 2019 a 2021 escritos em inglês, português ou espanhol como fonte de estudos e por abordarem a questão norteadora da revisão. As pesquisas demonstraram o surgimento de um círculo vicioso em que, com o estresse do cenário atual a população desrespeita as medidas de isolamento adotadas, resultando em um aumento do número de casos e superlotação nos hospitais. Com isso, têm-se uma sobrecarga física e emocional sobre os profissionais de saúde que encaram a luta diária contra o vírus.
\end{abstract}

Palavras-chave: Pandemia; Saúde mental; Profissionais de saúde; Problemas sociais.

\begin{abstract}
The present work aims to reflect on the mental health of professionals working in the front line and, with this, to raise discussions about the emotional impacts on their lives and on society as a result of the COVID-19 pandemic. The study comprises an integrative literature review. Searches for publications were carried out in four bibliographic databases - PubMed, Virtual Health Library (VHL), Scientific Electronic Library Online (SciELO) and Latin American and Caribbean Literature in Health Sciences (LILACS). 16 articles published between the years 2019 to 2021 were selected, written in English, Portuguese or Spanish as a source of studies and for addressing the guiding question of the review. Research has shown the emergence of a vicious circle in which, with the stress of the current scenario, the population disregards the isolation measures adopted, resulting in an increase in the number of cases and
\end{abstract}


overcrowding in hospitals. With this, there is a physical and emotional burden on health professionals who face the daily fight against the virus.

Keywords: Pandemic; Mental health; Health professionals; Social problems.

\section{Resumen}

El presente trabajo tiene como objetivo reflexionar sobre la salud mental de los profesionales que trabajan en primera línea y, con ello, plantear debates sobre los impactos emocionales en sus vidas y en la sociedad como consecuencia de la pandemia COVID-19. El estudio comprende una revisión integradora de la literatura. Las búsquedas de publicaciones se realizaron en cuatro bases de datos bibliográficas: PubMed, Biblioteca Virtual en Salud (BVS), Biblioteca Electrónica Científica en Línea (SciELO) y Literatura Latinoamericana y del Caribe en Ciencias de la Salud (LILACS). Se seleccionaron 16 artículos publicados entre los años 2019 a 2021, escritos en inglés, portugués o español como fuente de estudios y para abordar la pregunta orientadora de la revisión. Las investigaciones han demostrado la emergencia de un círculo vicioso en el que, con el estrés del escenario actual, la población desconoce las medidas de aislamiento adoptadas, lo que se traduce en un aumento del número de casos y hacinamiento en los hospitales. Con esto, existe una carga física y emocional para los profesionales de la salud que enfrentan la lucha diaria contra el virus.

Palabras clave: Pandemia; Salud mental; Profesionales de la salud; Problemas sociales.

\section{Introdução}

Em dezembro de 2019 foi identificado na cidade de Wuhan, na China, uma nova doença causada pela variação do vírus SARS-COV-2, a qual foi intitulada abreviadamente de COVID-19. Devido sua transmissão por meio de gotículas emitidas pelo sistema respiratório ao tossir ou espirrar, o vírus rapidamente se disseminou por diversos países, o que levou a Organização Mundial da Saúde (OMS) em 11 de março de 2020 a declarar a doença como uma pandemia (Ali \& Alharbi, 2020; Barroso, Souza, Bregalda, Lancman \& Costa, 2020).

Ademais, os diferentes níveis de infecção que abrangem desde assintomáticos a síndromes respiratórias agudas graves (SARS), dificultam a adoção de tratamento e as tentativas de controle da disseminação. Com isso, a quarentena e as medidas de isolamento social tornaram-se fundamentais para atenuar o contágio, uma vez que mantendo as pessoas distantes umas das outras o risco de contaminação diminui e, consequentemente, a procura por serviços de saúde e o número de óbitos também. No entanto, os estudos realizados por Souza et.al (2021), observaram que nesse período os níveis de ansiedade (19,4\%), depressão $(21,5 \%)$ e estresse rigoroso $(21,5 \%)$ elevaram-se exponencialmente.

Nesse sentido, os transtornos também se aplicam aos profissionais que atuam no combate a essa doença, já que as pressões de diversos setores e a responsabilidade por várias vidas afetam diretamente as condições ideais para um ambiente de trabalho saudável. Notou-se prejuízos na qualidade de sono e, consequentemente na qualidade de vida, visto que muitos já possuíam comorbidades prévias, principalmente psiquiátricas (Brito-Marques, Franco, Brito-Marques, Martinez \& Prado, 2021). Assim, a OMS, em uso de suas atribuições, constituiu um guia para orientar cuidados à saúde mental de diversos grupos, incluindo os profissionais de saúde (Rodríguez \& Sánchez, 2020; Walton, Murray \& Christian, 2020)

Diante desse cenário, para esses trabalhadores, o estresse e a pressão de lidar com o ofício, acrescido do risco de adoecimento, provocam severos problemas de saúde mental, aumentando o turnouver e a incidência da síndrome de Burnout (Meireles, 2020).

Dito isso, o presente trabalho visa refletir acerca da situação de saúde mental dos profissionais de saúde atuantes na linha de frente no enfrentamento a COVID-19 e, com isso, angariar discussões sobre os impactos da pandemia na vida dos mesmos.

\section{Metodologia}

O estudo compreende uma revisão integrativa da literatura, ou seja, com definição a priori da identificação do problema, seguido da busca, análise e síntese de dados de maneira sistemática e abrangente. Foi realizada a categorização, 
ordenação e sumarização dos resultados de forma descritiva, pontuando as questões mais significantes. A detecção das publicações, assim como a seleção e a classificação das mesmas foram seguidas de forma padronizada, assegurando o rigor metodológico da presente revisão (Broome, 2000).

As buscas dos estudos foram realizadas em quatro bases de dados bibliográficas - PubMed, Biblioteca Virtual em Saúde (BVS), Scientific Eletronic Library Online (SciELO) e Literatura Latino-Americana e do Caribe em Ciências da Saúde (LILACS) - e em pesquisa documental exploratória, na análise e interpretação de publicações em revistas científicas e manuais eletrônicos. Foram selecionados artigos publicados nos anos de 2019 a 2021 escritos em inglês, português ou espanhol. Para a realização da busca, foram utilizados os seguintes Descritores em Ciências da Saúde (DECS): "Pandemia"; "Saúde Mental"; "Profissionais da Saúde"; "Problemas Sociais" e utilizado combinações dos mesmos com o auxílio do operador lógico booleano "AND". Na pesquisa, foram inicialmente identificados 331 artigos científicos nas bases supracitadas, para a leitura exploratória do tema, desses, foram examinados 40 artigos nos idiomas mencionados anteriormente, disponíveis online na íntegra.

Depois da análise desses materiais, 16 foram inclusos como fonte de estudos e por abordarem a questão norteadora dos impactos emocionais gerados pela pandemia aos profissionais de saúde. Os critérios de exclusão foram artigos que não atendiam à temática, textos não disponíveis na íntegra, referências duplicadas e revisões de literatura.

As limitações encontradas foram alguns estudos baseados em análises transversais e correlações, não havendo a compreensão clara sobre as causas e os impactos tardios da pandemia na saúde mental dos trabalhadores. Além disso, houveram dificuldades diferentes em cada país, divergindo as medidas de abordagem entre eles. Uma pequena parcela dos profissionais de saúde participou desses estudos e, a falta de acesso à internet em alguns locais unida a sobrecarga de serviço propiciaram essa situação (Gloster et al., 2020).

\section{Resultados}

As pandemias habitualmente acarretam mortes em massa em pouco tempo, o que traz inferências psicológicas variadas (Crepaldi, Schmidt, Noal, Boze \& Gabarra, 2020; Rodríguez \& Sánchez, 2020). Segundo o Ministério da Saúde, do início do primeiro caso confirmado até abril de 2021 o Brasil já apresentava 14.308.215 casos confirmados e 389.492 óbitos. Com a necessidade constante de distanciamento social e o cenário assustador de mortes, foi ocasionado: estresse, pânico, insônia, ociosidade, medo, ansiedade, angústia, solidão, entre outros sentimentos, que são reações negativas para a saúde mental da população. Ademais, são vivenciados de forma individual e singular (Crepaldi, Schmidt, Noal, Boze \& Gabarra, 2020).

Com o avanço do Covid-19 entre as fronteiras mundiais, as sociedades amedrontadas e inseguras foram submetidas ao desconhecido e afastadas das interações face a face com membros das suas redes socioafetivas (Crepaldi, Schmidt, Noal, Boze \& Gabarra, 2020). Além disso, a ausência de informações consolidadas sobre o tratamento e a demora da aplicação das vacinas já distribuídas no Brasil, por exemplo, corroboram com a potencialização de emoções irracionais no cenário da pandemia (Crepaldi, Schmidt, Noal, Boze \& Gabarra, 2020; Rodríguez \& Sánchez, 2020). De acordo com "vacinômetro" do Ministério da Saúde foram distribuídas 57.866.738 vacinas no território nacional, sendo aplicadas na população apenas 37.571 .950 doses.

Por outro lado, encontram-se os profissionais de saúde que abdicam das interações com sua rede de familiares/amigos e além disso, convivem há um ano com a responsabilidade de ser a linha de frente no combate ao coronavírus. Segundo levantamento realizado pela Associação Médica Brasileira (AMB) em 826 instituições de saúde incluindo rede pública e privada, 95\% relataram problemas com fornecimento de máscaras N95, óculos, face shield, avental impermeável, foram relatados até mesmo a falta de luvas e álcool em gel, fomentando assim o receio desses profissionais que estão diariamente tratando de pessoas contaminadas (Associação Médica Brasileira [AMB], 2020) 
Ademais, a falta desses insumos para a realização de suas funções corrobora com os dados apresentados pela Secretaria de Vigilância em Saúde em que demonstram elevado crescimento no número de profissionais infectados que necessitam de hospitalização. Dentre esses, houveram 1230 casos notificados de SARS internados dos quais 22,5\% evoluíram para óbito, sendo a maioria (95,7\%) por covid-19 (Ministério da Saúde [MS], 2021).

Como registrado pela geolocalização do inloco (Gráfico 1), durante os meses da pandemia do Covid-19 os índices do isolamento social oscilavam e com sua baixa, havia mais propagação do vírus. Com isso, houve um aumento do número de casos e consequente lotação dos hospitais, pois alguns pacientes evoluíam com sintomas moderados a graves e necessidade de atendimento, essa situação sustentada ao longo dos meses culminou com profissionais da saúde exaustos emocional e fisicamente (Inloco, 2021).

Gráfico 1. Variação do índice de isolamento social no Brasil durante os meses de março de 2020 a fevereiro de 2021.

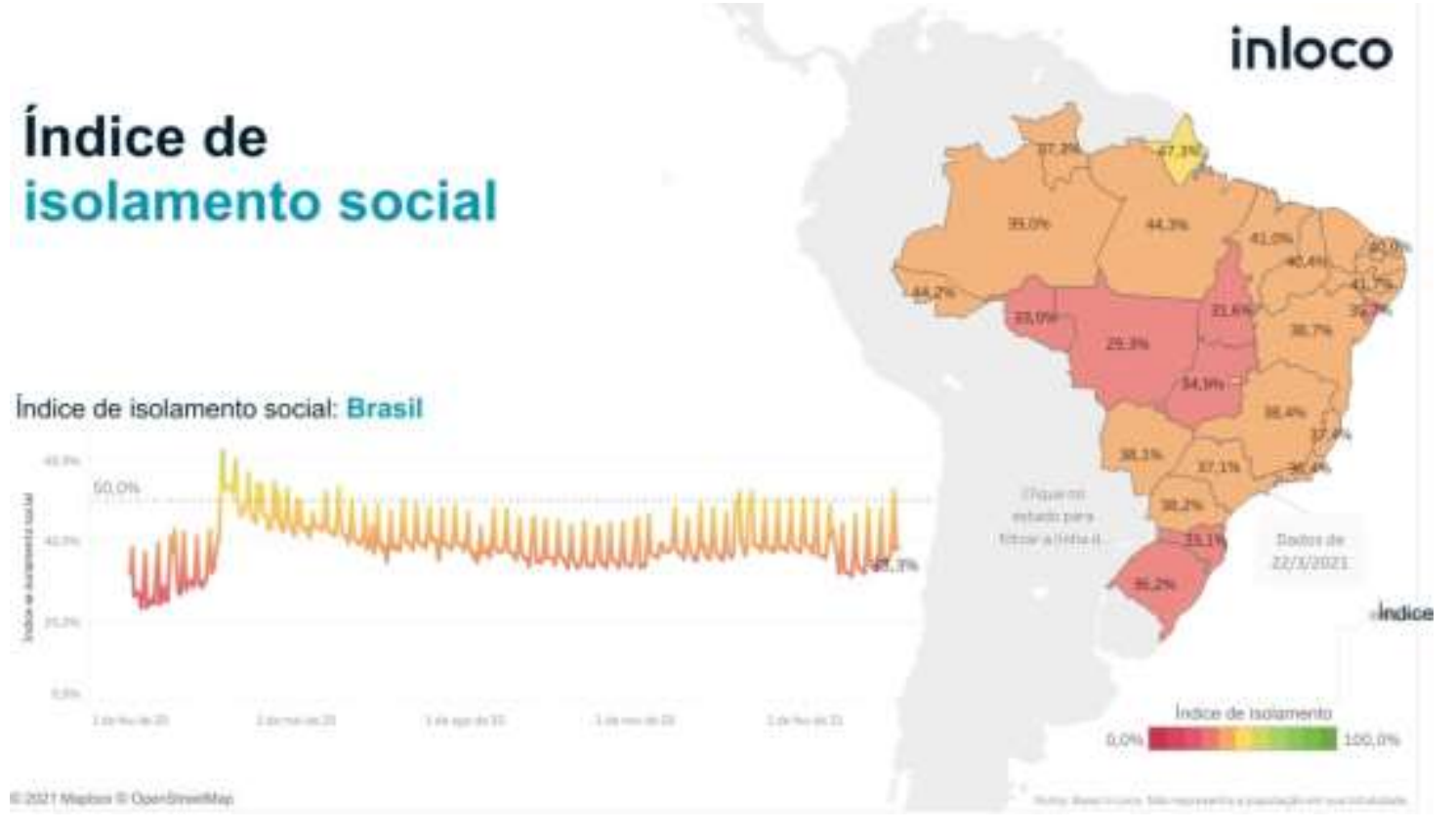

Fonte: Base In Loco. (2021). Mapa brasileiro da COVID-19. Disponível em: https://mapabrasileirodacovid.inloco.com.br/pt

Em países como o Equador cerca de 90\% da equipe médica e da enfermagem apresentaram Síndrome de Burnout moderada a grave, tais dados são preocupantes pois esse distúrbio pode evoluir com sintomas de ansiedade, depressão, uso de entorpecentes e suicídio (Inloco, 2021; Vinueza Veloz et al., 2020).

\section{Discussão}

Com a situação atípica gerada pela pandemia, a necessidade de implementar medidas de isolamento social para que houvesse reduções na disseminação do vírus se tornaram aparentes, permitindo, assim, estudos para o tratamento adequado dos pacientes que necessitarem de cuidados, além do desenvolvimento de vacinas e medicamentos que sejam eficazes contra a patologia. A atual conjuntura pandêmica remodelou os diversos segmentos sociais, impactou inúmeros setores, principalmente os relacionados diretamente com a saúde (Ministério da Saúde [MS], 2020).

Essa situação incomum na rotina dos cidadãos e os sentimentos de angustia e medo são refletidos e acentuam-se nos profissionais de saúde da linha de frente, uma vez que se expõem diariamente ao vírus, sofrem com a escassez de equipamentos de proteção individual, insalubridade, perca de colegas de trabalho decorrente do Covid-19. Além disso, há uma percepção do estigma social direcionado a eles pela alta probabilidade de estarem contaminados, isolamento da família e medo 
de contaminá-los, além da pressão e sobrecarga de trabalho (poucos funcionários, alta carga horária e pouco descanso) que gera esgotamento físico e psicológico, associada à alta demanda de suas habilidades cognitivas e emocionais (Stein, RoyByrne \& Salomon, 2021).

Devido a isso, as reações agudas de estresse que normalmente desaparecem em algumas semanas, viraram sinais permanentes na vida dos profissionais que estão submersos nesse contexto, aumentando a sobrecarga e a insatisfação com o ofício e influenciando indiretamente no tratamento de pacientes internados (Faro et al., 2020).

Contudo, os profissionais da saúde recebem homenagens nas redes sociais e há uma idealização de heroísmo do seu trabalho, mas a cobrança por produtividade, falta de treinamento para lidar com crises (por profissionais que passaram experiências semelhantes), a escassez de suporte psicológico e as poucas evidências cientificas confiáveis tornara-os, por vezes, mártires que, escolheram uma profissão que visa o cuidado (Correia, Ramos \& Bahten, 2020).

Dessa forma, foi criado um círculo vicioso em que, com a população cada dia mais estressada por todo o cenário e medidas de quarentena adotadas, resultando em desrespeito ao isolamento social, passa a buscar interações teoricamente proibidas nesse momento delicado. Como resposta dessa interação, tem-se o aumento do número de casos e a superlotação dos hospitais, acarretando mais estresse para os profissionais de saúde que encaram a luta diária contra o vírus (Medeiros, 2020).

\section{Considerações Finais}

Diante do exposto, é imprescindível que as organizações e instituições forneçam acesso rápido a aconselhamento, atendimento psiquiátrico e contingência para afastamento do trabalho àquele profissional que necessitar. Além disso, observar o cumprimento das jornadas de trabalho e das folgas implementadas que são essenciais para influenciar o bem-estar da equipe.

Ademais, é crucial a expansão da consciência crítica por parte da sociedade e a adaptação às medidas de isolamento, apesar de a vacinação já se mostrar promissora e efetiva. No Brasil, entretanto, a morosidade vacinal tem prejudicado a visibiliddade dos resultados e a quarentena ainda tem se mostrado uma das medidas mais eficazes contra a transmissão do novo coronavirus.

\section{Referências}

Ali, I., \& Alharbi, O. M. (2020). COVID-19: Disease, management, treatment, and social impact. Science of the total Environment, $728,138861$.

Associação Médica Brasileira. (2020). Falta EPIs no Brasil Inteiro!!! https://amb.org.br/noticias/falta-epis-no-brasil-inteiro/

Barroso, B. I. D. L., Souza, M. B. C. A. D., Bregalda, M. M., Lancman, S., \& Costa, V. B. B. D. (2020). A saúde do trabalhador em tempos de COVID-19: reflexões sobre saúde, segurança e terapia ocupacional. Cadernos Brasileiros de Terapia Ocupacional, 28(3), 1093-1102.

Brito-Marques, J. M. D. A. M., Franco, C. M. R., Brito-Marques, P. R. D., Martinez, S. C. G., \& Prado, G. F. D. (2021). Impacto da pandemia COVID-19 na qualidade do sono dos médicos no Brasil. Arquivos de Neuro-Psiquiatria, 79(2), 149-155.

Broome, M. E. (2000). Integrative literature reviews for the development of concepts. Concept development in nursing: foundations, techniques and applications. Philadelphia (USA): WB Saunders Company, 231-50.

Correia, M. I. T. D., Ramos, R. F., \& Bahten, L. C. V. (2020). Os cirurgiões e a pandemia do COVID-19. Revista do Colégio Brasileiro de Cirurgióes, 47.

Crepaldi, M. A., Schmidt, B., Noal, D. D. S., Bolze, S. D. A., \& Gabarra, L. M. (2020). Terminalidade, morte e luto na pandemia de COVID-19: demandas psicológicas emergentes e implicações práticas. Estudos de Psicologia (Campinas), 37.

Faro, A., Bahiano, M. D. A., Nakano, T. D. C., Reis, C., Silva, B. F. P. D., \& Vitti, L. S. (2020). COVID-19 e saúde mental: a emergência do cuidado. Estudos de Psicologia (Campinas), 37.

Gloster, A. T., Lamnisos, D., Lubenko, J., Presti, G., Squatrito, V., Constantinou, M., \& Karekla, M. (2020). Impact of COVID-19 pandemic on mental health: An international study. PloS one, 15(12), e0244809.

Horta, R. L., Camargo, E. G., Barbosa, M. L. L., Lantin, P. J. S., Sette, T. G., Lucini, T. C. G., \& Lutzky, B. A. (2021). O estresse e a saúde mental de profissionais da linha de frente da COVID-19 em hospital geral. Jornal Brasileiro de Psiquiatria, 70(1), 30-38.

Inloco. (2021). Mapa brasileiro da COVID-19. https://mapabrasileirodacovid.inloco.com.br/pt/ 
Research, Society and Development, v. 10, n. 6, e11610615516, 2021

(CC BY 4.0) | ISSN 2525-3409 | DOI: http://dx.doi.org/10.33448/rsd-v10i6.15516

Lindemann, I. L., Simonetti, A. B., Amaral, C. P. D., Riffel, R. T., Simon, T. T., Stobbe, J. C., \& Acrani, G. O. (2021). Percepção do medo de ser contaminado pelo novo coronavírus. Jornal Brasileiro de Psiquiatria, 70(1), 3-11.

Medeiros, E. A. S. (2020). Desafios para o enfrentamento da pandemia covid-19 em hospitais universitários. Revista Paulista de Pediatria, 38.

Meireles, G. (2020). Profissionais da linha de frente encaram desafios de saúde mental na pandemia. https://www.medicina.ufmg.br/profissionais-da-linha-defrente-encaram-desafios-de-saude-mental-na-pandemia/

Ministério da Saúde. (2020). Boletim Epidemiológico COE COVID-19: Semana Epidemiológica 15 (5-10/04/2020) https://portalarquivos.saude.gov.br/images/pdf/2020/April/09/be-covid-08-final-2.pdf

Ministério da Saúde. (2021). Boletim Epidemiológico Especial Doença pelo Coronavírus COVID-19: Semana Epidemiológica 14 (4/4 a 10/4/2021). https://www.gov.br/saude/pt-br/media/pdf/2021/abril/16/boletim_epidemiologico_covid_58-1.pdf

Rodríguez, B. O., \& Sánchez, T. L. (2020). The Psychosocial Impact of COVID-19 on health care workers. International braz j urol, 46, 195-200.

Souza, A. S. R., Souza, G. F. A., Souza, G. A., Cordeiro, A. L. N., Praciano, G. A. F., Alves, A. C. D. S., \& Souza, M. B. R. (2021). Factors associated with stress, anxiety, and depression during social distancing in Brazil. Revista de Saúde Pública, $55,5$.

Stein, M. B., Roy-Byrne, P. P. \& Salomon, D. (2021). Covid-19: Doença psiquiátrica. https://www.uptodate.com/contents/covid-19-psychiatricillness? source=history_widget

Vinueza Veloz, A. F., Aldaz Pachacama, N. R., Mera Segovia, C. M., Pino Vaca, D. P., Tapia Veloz, E. C., \& Vinueza Veloz, M. F. (2020). Síndrome de Burnout en médicos/as y enfermeros/as ecuatorianos durante la pandemia de COVID-19. Revista de la Asociación Española de Especialistas en Medicina del Trabajo, 29(4), 330-339.

Walton, M., Murray, E., \& Christian, M. D. (2020). Mental health care for medical staff and affiliated healthcare workers during the COVID-19 pandemic. European Heart Journal: Acute Cardiovascular Care, 9(3), 241-247. 